\title{
Prevalence and predictors of involuntary psychiatric hospital admissions in Ontario, Canada: a population-based linked administrative database study
}

Michael Lebenbaum, Maria Chiu, Simone Vigod and Paul Kurdyak

\section{Background}

Involuntary admissions to psychiatric hospitals are common; however, research examining the trends in prevalence over time and predictors is limited.

\section{Aims}

To examine trends in prevalence and risk factors for involuntary admissions in Ontario, Canada.

\section{Method}

We conducted an analysis of all mental health bed admissions from 2009 to 2013 and assessed the association between patient sociodemographics, service utilisation, pathway to care and severity characteristics for involuntary admissions using a modified Poisson regression.

\section{Results}

We found a high and increasing prevalence of involuntary admissions (70.7\% in 2009, $77.1 \%$ in $2013,74.1 \%$ overall). Individuals with police contact in the prior week (risk ratio $(R R)=1.20$ ) and immigrants both experienced greater likelihood of being involuntarily admitted, regardless of control for other characteristics $(\mathrm{RR}=1.07)$ (both $P<0.0001)$.

\section{Conclusions}

We identified numerous modifiable and non-modifiable risk factors for involuntary admissions. The prevalence of involuntary admissions was high, linearly increasing over time.

\section{Declaration of interest}

The authors have completed the ICMJE Form for Disclosure of Potential Conflicts of Interest and none were reported. This study was conducted using funding entirely from public sources. P.K. has received operational support via an Ontario Ministry of Health and Long-Term Care (MOHLTC) Health Services Research Fund Capacity Award to support this project. The Institute for Clinical Evaluative Sciences (ICES) is funded by the Ontario MOHLTC. The study results and conclusions are those of the authors, and should not be attributed to any of the funding agencies or sponsoring agencies. No endorsement by ICES or the Ontario MOHLTC is intended or should be inferred. All decisions regarding study design, publication, and data analysis were made independent of the funding agencies.

\section{Copyright and usage}

(c) The Royal College of Psychiatrists 2018. This is an Open Access article, distributed under the terms of the creative commons Attribution-NonCommercial-NoDerivatives licence (http:// creativecommons.org/licenses/by-nc-nd/4.0/), which permits non-commercial re-use, distribution, and reproduction in any medium, provided the original work is unaltered and is properly cited. The written permission of Cambridge University Press must be obtained for commercial re-use or in order to create a derivative work.
Involuntary psychiatric hospital admissions generally occur when an individual with mental illness is admitted to hospital against their will owing to a perceived imminent danger to the individual or others and unwillingness to remain in hospital voluntarily. These admissions are not desirable, because they can be disruptive to the patient-provider relationship and have a negative impact on the patient's perception of their care at the time of the admission and afterwards. ${ }^{1-3}$ Although there is variability across jurisdictions, involuntary admissions are common and increasing in high-income countries, including several European countries such as the UK, ${ }^{4,5}$ The Netherlands ${ }^{5,6}$ and Germany. ${ }^{5}$ There are few studies investigating trends in involuntary admissions over time in other jurisdictions.

Involuntary admission to hospital may, to some extent, be an avoidable event if care provided in community settings mitigates psychiatric crises that precipitate involuntary admissions. Studies mostly from Europe have shown that, in addition to these servicerelated factors, risk factors for involuntary admissions include patient sociodemographic characteristics such as being an immigrant or ethnic minority, and clinical severity variables such as a diagnosis of a psychotic disorder. ${ }^{7-11}$ Studies from other jurisdictions are largely from the USA and emphasise the importance of available mental health resources. ${ }^{12,13}$ In Canada, the most recent country-wide prevalence estimate of involuntary admissions
(25\%) was in the late 1970s. ${ }^{14}$ One recent study among 200 patients with first-episode psychosis at four sites in Ontario had a prevalence of involuntary admissions of $68.6 \%,{ }^{15}$ suggesting a large increase in prevalence. However, no recent population-based Canadian study has examined prevalence of involuntary admissions among all patients. ${ }^{16,17}$ Furthermore, existing Canadian studies have examined only a very limited set of characteristics of involuntarily admitted patients. ${ }^{18-21}$ This is consistent with many international studies, which are often small in size, based on one or a few sites, and examine only a limited number of risk factors. The aims of this study were to determine the trends in prevalence of involuntary admissions in a large population-based North American sample covering the whole Province of Ontario, Canada (population $\sim 14$ million), and to examine the independent risk factors that predict involuntary admission status in this jurisdiction.

\section{Methods}

\section{Study design, setting, and participants}

We examined the prevalence and risk factors of involuntary admissions among all patients admitted to mental health and addictions (MHA) beds in Ontario, Canada. Annual prevalence was examined 
from fiscal year (April 1 to March 31) 2009 to 2013, and risk factors for involuntary admission were examined for all years pooled. The index date was defined as the admission date, and risk factors were measured at or up to 5 years prior to index.

We included adults aged 16-105 years at index admission date who were Ontario residents with valid health card numbers, owing to the requirement to link across databases. We excluded any informal (decision to admit was made by a substitute decision maker) and forensic admissions, as these patients are ineligible for voluntary or involuntary admissions. We excluded any patients without a linked emergency department record immediately preceding the admission date, given that elective admissions are a different population and a number of previous studies have taken place in the psychiatric emergency department. For individuals with more than one admission to hospital during the study period, we included one random admission. We also excluded individuals without healthcare eligibility at 1 year prior to index, given that a number of risk factors were measured in the year prior to admission.

\section{Data sources}

We used the Ontario Mental Health Reporting System (OMHRS) to identify our study population of patients who had been admitted to a mental health bed. The OMHRS database uses the Resident Assessment Instrument - Mental Health (RAI-MH), a standardised assessment instrument that captures a number of sociodemographic characteristics, diagnoses according to the DSM-IV and clinical assessments.

To ascertain sociodemographic information and other patientlevel factors and service use, we linked the OMHRS database to other administrative health databases at the Institute for Clinical Evaluative Sciences, including the Canadian Institute for Health Information (CIHI) hospital Discharge Abstract Database (DAD), the Ontario Health Insurance Program (OHIP) physician claims database, the National Ambulatory Care Reporting System (NACRS) emergency department database, the Registered Persons Database (RPDB) and the Immigration Refugees and Citizenship Canada Permanent Resident (IRCC-PR) database.

\section{Involuntary admissions}

Involuntary admissions are defined within OMHRS as a patient status of either Form 1 or Form 3 at admission. Involuntary admissions in Ontario in most cases begin in the emergency department, where a physician will complete an Application for Psychiatric Assessment ('Form 1'); this is an involuntary hold that allows the patient to be detained and examined for up to $72 \mathrm{~h}$ in a psychiatric hospital. $^{22}$ In some cases, a Form 3 is subsequently completed to involuntarily admit the patient for up to 2 weeks. The Form 3 is completed by a psychiatrist, who must be a different physician than the physician who completed the Form 1.

\section{Predictors of involuntary admissions}

\section{Sociodemographic variables}

Patient sociodemographic characteristics included age (16-24, 25-44, $45-64,65+)$, gender, area-level income quintiles identified with the use of the RPDB, housing status (homeless/private/non-private) identified within OMHRS, and immigration status identified within the IRCC-PR and categorised as immigrant or long-term resident (including non-immigrants or immigrants migrating prior to 1985).

\section{Prior mental health service use}

Past service use included whether they had had a mental health admission, mental health emergency department visit, psychiatrist visit, or a mental health ${ }^{23}$ or non-mental health family physician visit within the past year (definitions are listed in supplementary Table 1; available at https://doi.org/10.1192/bjo.2017.4). In addition, we examined whether they had any involuntary records within the past 5 years, including admissions or physician billings for involuntary assessments.

\section{Pathway to care variables}

The pathway to care was defined as care received or contacts acquired during the week prior to and including the day of the emergency department visit. This included contact with police, outpatient visits to a psychiatrist, and mental health and non-mental health out-patient visits to family physicians. Physician visits were identified in the OHIP database, and mental health visits to family physicians was identified with a validated algorithm. ${ }^{23}$ We also examined whether the patient arrived at the emergency department via ambulance or had a transfer from a medical bed prior to admission to the mental health bed.

\section{Clinical variables}

We included several clinical measures of patient severity. We assessed the primary diagnoses, which included schizophrenia or psychosis, mood disorders, anxiety disorders, dementia and all other psychiatric diagnoses. We examined whether they had any indication of a substance misuse disorder or a personality disorder. We identified emergency department visits that had indication of self-harm. Specific codes for each diagnosis are listed in supplementary Table 1 . Triage status was measured on a five-point scale and combined into low (suicidal/depressed or other milder psychiatric complaints and not agitated), medium (acute psychosis and/or suicidal) and high triage (patients experiencing acute psychosis/ extreme agitation). ${ }^{24}$ We also included whether they had any comorbidities included in the Charlson comorbidity index as assessed in the year prior to admission.

We also examined a number of InterRAI rating scales at admission, including the Severity of Self-Harm (SOS), Risk of Harm to Others (RHO) and Self-Care Index (SCI), which are all directly related to the criteria for involuntary admissions and included three symptom scales, the Positive Symptoms Scale (PSS), Mania Rating Scale (MRS) and Depression Rating Scale (DRS). ${ }^{25}$ The SOS, RHO and SCI are each based on predictive risk algorithms with combinations of inputs based on individual symptoms, past behaviour, or other symptom scales. ${ }^{25}$ Each of the PSS, MRS and DRS were based on items assessing the frequency of symptoms in the past 3 days as: 0 (not exhibited in past 3 days), 1 (not exhibited in the past 3 days but reported to be present), 2 (exhibited on 1-2 of the past 3 days), or 3 (exhibited daily). Each scale aggregated items to different numbers of points based on the number of symptoms and the points assigned to each frequency of occurrence. The range and list of symptoms included in the calculation of each scale is shown in supplementary Table 2 .

\section{Statistical methods}

We examined the prevalence of involuntary admissions overall and by year. We assessed the trend over time by fitting a linear time trend across the 5-year study period using a modified Poisson regression.

We estimated the prevalence of each risk factor for both voluntary and involuntary patients. We used a modified Poisson regression to determine the unadjusted and multivariable adjusted association between each risk factor and involuntary admissions. All variables were included in the multivariable model regardless of significance. Patients with missing data were excluded from analyses, except for individuals with missing income. All analyses were conducted in SAS Enterprise Guide 6.1. 


\section{Ethics committee approval}

Ethics approval for this study was obtained from the Research Ethics Board at Sunnybrook Health Sciences Centre in Toronto, Ontario.

\section{Results}

A total of 250773 admissions were identified between fiscal years 2009-2013 among adults living in Ontario with valid health card numbers. We excluded informal or forensic admissions $(n=$ 7214), individuals who had voluntary admissions that subsequently were coded as involuntary $(N=676)$, individuals without eligibility for healthcare a year prior to index $(N=2831)$, and individuals who lacked linked emergency department records $(n=39589)$. Of the remaining 200463 admissions, we kept one random admission per individual ( $N=84948$ admissions excluded), which resulted in a final sample size of 115515 patients admitted to hospital.

Among the 115515 individuals who met inclusion criteria, 85 607 (74.1\%) were involuntarily admitted. The prevalence of involuntary admissions significantly increased from $70.7 \%$ in 2009 to $77.1 \%$ in $2013(\mathrm{RR}=1.021$ per year, 95\% CI 1.019-1.023; $P<$ 0.0001 ) (Fig. 1). 28726 (33.6\%) of individuals who were involuntarily admitted were released within $72 \mathrm{~h}$ of admission. Additional descriptive characteristics for patients by involuntary status are shown in Table 1.

Sociodemographic, utilisation, pathway to care and severity characteristics are summarised by involuntary status in Table 2, along with unadjusted and adjusted associations from modified Poisson regression. Most variables were complete, except for 645 individuals missing triage status, 764 individuals with missing income and 8458 individuals missing SOS and SCI.

Almost all variables were associated with involuntary admission to hospital in unadjusted analyses (Table 2). The strongest factors associated with increased likelihood for involuntary admission were past week police contact $(\mathrm{RR}=1.36,95 \% \mathrm{CI} 1.36-1.37)$ and severity variables including high-acuity triage status $(\mathrm{RR}=1.55$, 95\% CI 1.51-1.58) and medium triage status $(\mathrm{RR}=1.30,95 \% \mathrm{CI}$ $1.27-1.33) v$. low acuity, self-harm identified in the emergency department contact preceding the admission $(\mathrm{RR}=1.17,95 \% \mathrm{CI}$ 1.16-1.18). Not having a psychotic disorder ( $R R=0.83-0.93$, depending on the diagnosis) and having a mental health visit in the week preceding admission with either a family physician (RR $=0.89,95 \%$ CI $0.88-0.89)$ or a psychiatrist $(\mathrm{RR}=0.94,95 \% \mathrm{CI}$ $0.93-0.95)$ were protective. These relationships were all maintained, although slightly attenuated, in the adjusted models (Table 2). Several other variables were also significantly associated with

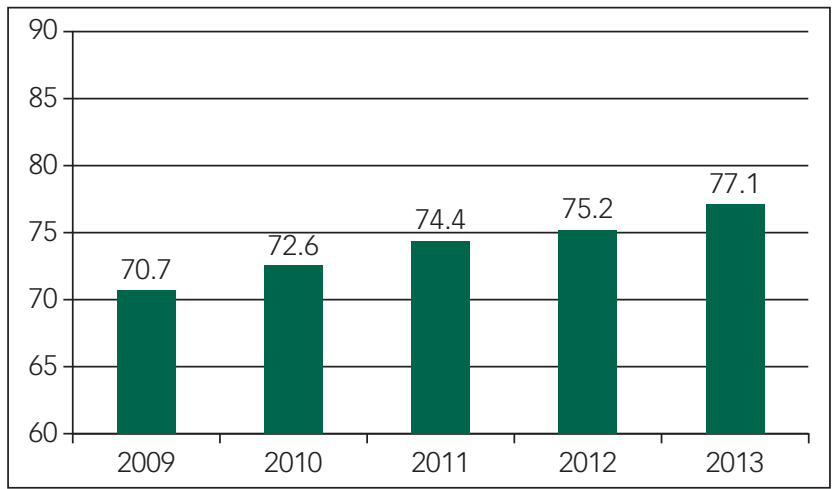

Fig. 1 Trends over time in the prevalence of involuntary admissions within Ontario, Canada.
Table 1 Descriptive characteristics for patients who are involuntary or voluntary at admission

\begin{tabular}{|c|c|c|}
\hline & $\begin{array}{l}\text { Involuntary } \\
(N=85607)\end{array}$ & $\begin{array}{l}\text { Voluntary } \\
(N=29908)\end{array}$ \\
\hline & $\%$ & $\%$ \\
\hline Percentage of total & 74.1 & 25.9 \\
\hline \multicolumn{3}{|l|}{ Reason for admission } \\
\hline Only threat to others & 5.5 & 1.8 \\
\hline Only threat to self & 40.3 & 33.6 \\
\hline $\begin{array}{l}\text { Only inability to care for self due to mental } \\
\text { illness }\end{array}$ & 13.0 & 12.8 \\
\hline More than one of the above & 29.1 & 15.1 \\
\hline \multicolumn{3}{|l|}{ Type of hospital } \\
\hline Psychiatric & 10.0 & 13.5 \\
\hline General & 90.0 & 86.5 \\
\hline \multicolumn{3}{|l|}{ Status at admission } \\
\hline Form 1 & 73.5 & NA \\
\hline Form 3 & 26.5 & NA \\
\hline \multicolumn{3}{|c|}{ Status at initial assessment (Up to $72 \mathrm{~h}$ post admission) } \\
\hline Released (LOS $\leq 72 \mathrm{~h})$ & 33.6 & 32.7 \\
\hline Form 1 & 20.8 & 1.4 \\
\hline Form 3 & 28.2 & 2.0 \\
\hline voluntary & 17.1 & 63.9 \\
\hline Other & 0.4 & 0.1 \\
\hline LOS, Length of Stay. & & \\
\hline
\end{tabular}

involuntary admission in the adjusted models. Notably, younger age (16-24) $(\mathrm{RR}=1.10,95 \mathrm{CI} 1.08-1.12)$, immigrant status $(\mathrm{RR}=$ $1.07,95 \%$ CI 1.06-1.08) and previous involuntary assessments/ admissions ( $\mathrm{RR}=1.09,95 \% \mathrm{CI} 1.08-1.10)$ all increased the likelihood of involuntary admission. Meanwhile, past year mental health visits to psychiatrists $(\mathrm{RR}=0.94,95 \%$ CI $0.93-0.95)$ were associated with a reduced likelihood of involuntary admissions. After adjusting for other variables, there was no association between any level of income and likelihood of involuntary admission, and a minimal increased likelihood among males $(\mathrm{RR}=1.02$, 95\% CI 1.01-1.02).

The unadjusted and adjusted associations between six clinical rating scales are shown in Fig. 2. Higher scores on all scales were associated with an increased risk of involuntary admission, except for the DRS, where higher scores were associated with a decreased risk of involuntary admission.

\section{Discussion}

In this large population-based study, we found a very high prevalence of involuntary psychiatric admissions - almost three-quarters of all hospital admissions. The prevalence has increased steadily over the years between 2009 and 2013, from 70.7 to $77.1 \%$. There were also significant risk factors for involuntary admission to hospital among sociodemographic, past service utilisation, pathway to care, and clinical severity characteristics. The sociodemographic factors included immigration status and young age, both of which increased involuntary admissions. Mental health service utilisation in the prior year was also a strong predictor, with physician contact reducing the likelihood of involuntary admission. Police contacts in the week prior to admission, a pathway to care variable, and a number of severity variables including triage status, self-harm and psychosis were also risk factors increasing the likelihood of involuntary admissions.

Given previous Ontario estimates of $11-12 \%$ in the late $1970 \mathrm{~s}^{14,26}$ and $19.3 \%$ in $1983,{ }^{16,17}$ our results show that there has been a dramatic four-fold increase in the prevalence of involuntary admissions, such that three out of four psychiatric admissions to hospital start as 
Table 2 Breakdown of patient characteristics by involuntary status, and unadjusted and multivariable logistic regression of association between each characteristics and involuntary status

\begin{tabular}{|c|c|c|c|c|c|c|}
\hline & \multirow{2}{*}{$\begin{array}{c}\text { Involuntary } \\
(N=85607) \\
\%\end{array}$} & \multirow{2}{*}{$\begin{array}{c}\text { Voluntary } \\
(N=29908) \\
\%\end{array}$} & \multicolumn{2}{|c|}{ Unadjusted analysis } & \multicolumn{2}{|c|}{ Multivariable analysis } \\
\hline & & & $\mathrm{RR}$ & $95 \% \mathrm{Cl}$ & $\mathrm{RR}$ & $95 \% \mathrm{Cl}$ \\
\hline \multicolumn{7}{|l|}{ Sociodemographic characteristics } \\
\hline \multicolumn{7}{|l|}{ Age category, years } \\
\hline $16-24$ & 22.2 & 16.3 & 1.16 & $(1.15,1.18)$ & 1.10 & $(1.08,1.12)$ \\
\hline $25-44$ & 36.6 & 36.2 & 1.09 & $(1.07,1.10)$ & 1.04 & $(1.02,1.05)$ \\
\hline $45-64$ & 31.2 & 34.3 & 1.05 & $(1.04,1.07)$ & 1.03 & $(1.02,1.05)$ \\
\hline $65+$ & 10.0 & 13.1 & Reference & & & \\
\hline Male & 52.6 & 44.5 & 1.09 & $(1.08,1.09)$ & 1.02 & $(1.01,1.02)$ \\
\hline \multicolumn{7}{|l|}{ Housing status } \\
\hline Homeless & 3.2 & 2.7 & 1.05 & $(1.03,1.07)$ & 0.98 & $(0.96,1.00)$ \\
\hline Non-private & 6.3 & 5.7 & 1.03 & $(1.02,1.04)$ & 0.99 & $(0.98,1.01)$ \\
\hline Private residence & 90.5 & 91.7 & Reference & & & \\
\hline \multicolumn{7}{|l|}{ Income quintile } \\
\hline Missing & 0.7 & 0.6 & 1.07 & $(1.03,1.11)$ & 1.01 & $(0.97,1.05)$ \\
\hline Quintile 1 & 29.8 & 27.8 & 1.05 & $(1.03,1.06)$ & 1.01 & $(1.00,1.02)$ \\
\hline Quintile 2 & 21.2 & 21.9 & 1.02 & $(1.01,1.03)$ & 1.00 & $(0.98,1.01)$ \\
\hline Quintile 3 & 17.8 & 17.6 & 1.03 & $(1.02,1.04)$ & 1.01 & $(1.00,1.02)$ \\
\hline Quintile 4 & 16.7 & 16.8 & 1.03 & $(1.01,1.04)$ & 1.01 & $(1.00,1.02)$ \\
\hline Quintile 5 & 13.9 & 15.3 & Reference & & & \\
\hline Immigrant & 11.6 & 7.7 & 1.11 & $(1.10,1.12)$ & 1.07 & $(1.06,1.08)$ \\
\hline \multicolumn{7}{|l|}{ Past service utilisation } \\
\hline Past 5 years involuntary record & 34.1 & 26.1 & 1.10 & $(1.09,1.11)$ & 1.09 & $(1.08,1.10)$ \\
\hline \multicolumn{7}{|l|}{ Past year utilisation: } \\
\hline MHA admission & 20.1 & 20.6 & 0.99 & $(0.98,1.00)$ & 0.97 & $(0.96,0.98)$ \\
\hline MHA ED visit & 28.5 & 31.6 & 0.96 & $(0.95,0.97)$ & 0.98 & $(0.97,0.98)$ \\
\hline Out-patient psychiatrist visit & 39.3 & 45.2 & 0.94 & $(0.93,0.95)$ & 0.94 & $(0.93,0.95)$ \\
\hline Out-patient MHA family physician visit & 53.4 & 60.6 & 0.93 & $(0.92,0.93)$ & 0.98 & $(0.97,0.99)$ \\
\hline Out-patient non-MHA family physician visit & 75.7 & 81.2 & 0.92 & $(0.92,0.93)$ & 0.99 & $(0.98,1.00)$ \\
\hline \multicolumn{7}{|l|}{ Pathway to care } \\
\hline Transfer from medical bed & 9.9 & 10.0 & 1.00 & $(0.98,1.01)$ & 0.93 & $(0.92,0.94)$ \\
\hline \multicolumn{7}{|l|}{ Police contact } \\
\hline Past week & 28.8 & 7.2 & 1.36 & $(1.36,1.37)$ & 1.20 & $(1.19,1.21)$ \\
\hline Any other contact & 13.2 & 12.7 & 1.11 & $(1.10,1.12)$ & 1.03 & $(1.02,1.05)$ \\
\hline Never & 58.0 & 80.1 & Reference & & & \\
\hline Arrival by ambulance & 30.0 & 20.9 & 1.12 & $(1.12,1.13)$ & 1.09 & $(1.08,1.10)$ \\
\hline \multicolumn{7}{|l|}{ Past week physician visit: } \\
\hline Psychiatrist visit & 20.1 & 24.1 & 0.94 & $(0.93,0.95)$ & 0.98 & $(0.97,0.99)$ \\
\hline Family physician MHA visit & 14.4 & 20.5 & 0.89 & $(0.88,0.89)$ & 0.95 & $(0.94,0.96)$ \\
\hline Family physician non-MHA-visit & 12.3 & 13.2 & 0.98 & $(0.97,0.99)$ & 1.01 & $(1.00,1.02)$ \\
\hline \multicolumn{7}{|l|}{ Severity } \\
\hline \multicolumn{7}{|l|}{ Primary diagnosis } \\
\hline Schizophrenia or psychotic disorder & 26.3 & 15.6 & Reference & & & \\
\hline Anxiety and adjustment disorders & 12.7 & 13.0 & 0.89 & $(0.88,0.90)$ & 0.92 & $(0.90,0.93)$ \\
\hline Mood disorders & 39.8 & 52.2 & 0.83 & $(0.82,0.83)$ & 0.89 & $(0.88,0.90)$ \\
\hline Dementia & 3.0 & 2.5 & 0.93 & $(0.91,0.95)$ & 1.02 & $(1.00,1.04)$ \\
\hline Other & 18.3 & 16.7 & 0.91 & $(0.91,0.92)$ & 0.92 & $(0.91,0.93)$ \\
\hline Any indication of a substance abuse disorder & 29.1 & 24.6 & 1.06 & $(1.05,1.07)$ & 1.01 & $(1.00,1.02)$ \\
\hline Any indication of a personality disorder & 13.9 & 14.1 & 1.00 & $(0.99,1.01)$ & 0.99 & $(0.98,1.00)$ \\
\hline ED self-harm & 11.4 & 5.7 & 1.17 & $(1.16,1.18)$ & 1.11 & $(1.10,1.12)$ \\
\hline Triage status & & & & & & \\
\hline High & 55.2 & 35.2 & 1.55 & $(1.51,1.58)$ & 1.39 & $(1.36,1.42)$ \\
\hline Medium & 40.2 & 53.0 & 1.30 & $(1.27,1.33)$ & 1.22 & $(1.19,1.24)$ \\
\hline Low & 4.6 & 11.8 & Reference & & & \\
\hline Any Charlson comorbidity & 4.5 & 6.2 & 0.91 & $(0.89,0.93)$ & 0.96 & $(0.94,0.98)$ \\
\hline InterRAI clinical rating scales ${ }^{a, b}$ & & & See Fig. 2 & & & \\
\hline Risk of Harm to Others (RHO) & 1.9 & 1.1 & NA & NA & NA & NA \\
\hline Severity of Self-Harm (SOS) & 2.6 & 2.2 & NA & NA & NA & NA \\
\hline Self-Care Index (SCI) & 1.8 & 1.3 & NA & NA & NA & NA \\
\hline Positive Symptoms Scale (PSS) & 2.0 & 1.2 & NA & NA & NA & NA \\
\hline Mania Rating Scale (MRS) & 2.7 & 1.6 & NA & NA & NA & NA \\
\hline Depression Rating Scale (DRS) & 2.9 & 3.0 & NA & NA & NA & NA \\
\hline
\end{tabular}

involuntary. Although the prevalence of involuntary admissions has increased, current patients likely spend much less time being detained involuntarily relative to patients who were involuntarily admitted prior to 1978. Prior to 1978, patients were admitted involuntarily for up to 1 month, while fewer than half of current patients with involuntary status spend more than 3 days with involuntary status, 


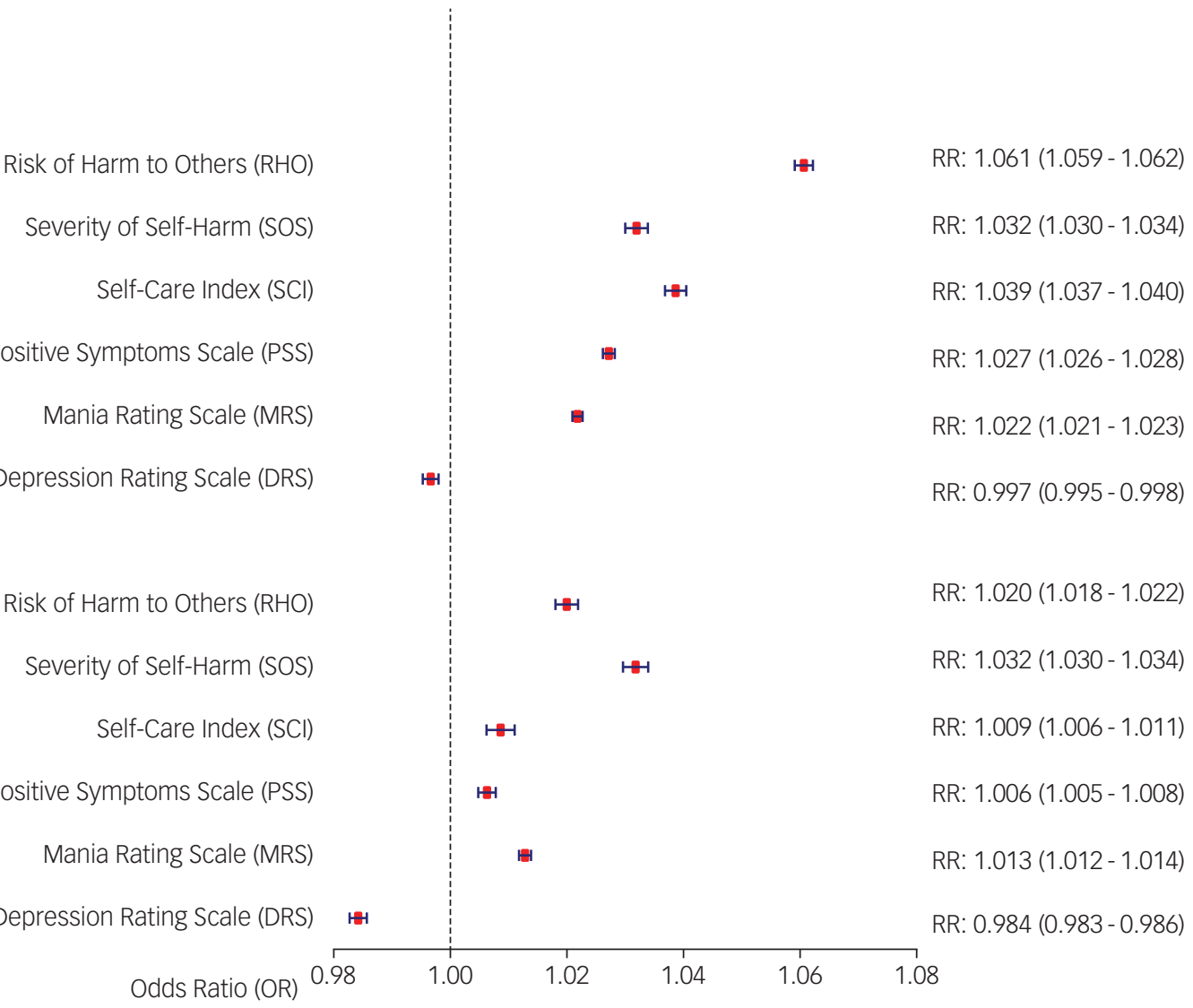

UNADJUSTED a Positive Symptoms Scale (PSS)

Mania Rating Scale (MRS)

Depression Rating Scale (DRS)

ADJUSTED ${ }^{b}$

Positive Symptoms Scale (PSS 
other administrative databases enabled an extensive examination of risk factors for involuntary admissions, including utilisation of different types of healthcare and immigration status. Lastly, the generalisability of the findings is enhanced by the use of a populationbased sample that collected data across all Ontario psychiatric beds from over 60 hospitals.

This study is not without limitations. First, although the OMHRS database collects data for all admissions to MHA beds, approximately $20 \%$ of MHA admissions are to non-MHA beds captured in the DAD and were not included, as the RAI-MH is not implemented in medical beds and most variables would be missing. Second, the exact pathway to care is not known; we used care received or contacts within the week prior to admission as proxies. Third, the symptom scales were recorded on the third day of the admission, reflecting the first 3 days, which may have attenuated their associations with involuntary admissions. However, symptoms likely only changed minimally over the course of 3 days, and having the rating scales completed 3 days after admission assures that the assessment of symptoms was not completed by the same individual who made the admission decision.

\section{International comparison of prevalence}

Reviews of mostly European countries, covering the period 19712000 , found that the prevalence ranged from a low of $1.0 \%$ in Spain to a high of $93 \%$ in some hospitals in Switzerland, with all other estimates less than $50 \%{ }^{35,36}$ Therefore, the prevalence found in our study is among the highest reported prevalences for a Western country. This considerable variation in prevalence across countries likely reflects variation in data collection and definitions, ${ }^{36}$ laws, ${ }^{36}$ psychiatric resources, ${ }^{5,37}$ cultures, ${ }^{38,39}$ and societal and physician attitudes towards involuntary admissions. ${ }^{40}$ A key contributing factor to the high prevalence in Ontario may be the low supply of psychiatric beds compared with both Western ${ }^{5}$ and Eastern European countries. ${ }^{37}$

Studies from East Asian countries have found even higher prevalence of involuntary admissions than found in Ontario, with one study in Korea finding a prevalence of $91 \%{ }^{38}$ In China, the prevalence is similarly high, with one study reporting that $42 \%$ of admissions to hospital were involuntary and $30 \%$ were partly voluntary. ${ }^{39}$ In both countries, this is potentially due to a more collectivist culture in which a family member is the deciding authority on the involuntary admission instead of the patient. ${ }^{39}$ However, this is not uniform across the Asian continent, with one of the lowest reported prevalences of $4.0 \%$ found in Taiwan. ${ }^{41}$ This is likely due to the strict criteria for involuntary admissions that only allow involuntary admissions of patients with psychosis and also requires non-compliance with treatment and dangerousness, and, although families are often involved, the patient still has to provide consent themselves. ${ }^{41}$

\section{International comparison of risk factors and implications}

We examined risk factors among an admitted cohort of patients, which allowed us to isolate which factors are specifically associated with involuntary status and not incorporated into the association factors that are associated with the decision to admit the patient or the decision to visit an emergency department. We found that youth aged 16-24 years were at a higher likelihood of involuntary admissions, regardless of control for other risk factors. This is not surprising, given the higher onset and burden of mental health conditions in this age group, and consistent access issues with long wait lists to receive care during a time of transition from youth to adult mental health services. ${ }^{42}$ Previous research on the role of age is conflicting, with studies showing increased likelihood with younger age $^{11}$ and older age, ${ }^{43}$ and others finding no associations with age. $^{7-9,20}$ The elevated likelihood of involuntary admissions among immigrants is a consistent finding in the UK and in other European countries, especially for immigrants or ethnic minorities from Caribbean, ${ }^{7,11}$ African ${ }^{7,11}$ or non-Western ${ }^{8}$ countries or ethnicities. Although some studies show that controlling for observable characteristics, including greater levels of psychosis and more adversarial pathways to care involving the justice system, explain these findings, ${ }^{8,10}$ our study is not the only study to find that these factors did not explain the excess likelihood of involuntary admissions. ${ }^{7}$ Patients with any diagnosis other than schizophrenia or psychosis were at lower likelihood of involuntary admissions, which is a common finding with these conditions. ${ }^{20,39,44}$ Psychosis is elevated among younger individuals and among immigrants, which together suggests that individuals with psychosis may be a key target group for future intervention studies which may help disparities among key demographic groups. Further research is required to understand which groups of immigrants are at excess likelihood of involuntary admission and explore possible explanations and potential interventions to address this disparity.

Our findings of increased likelihood with adversarial routes such as police contacts ${ }^{10,41,44}$ and decreased likelihood with physician contact $^{7}$ are consistent with the international literature. We also found independent associations between past year mental health visits and visits on the pathway to care, both with decreased likelihood of involuntary admissions. This suggests that potential interventions to reduce the likelihood of involuntary admission may involve increasing access to out-patient resources for patients with severe mental illness.

A wide variety of severity measures were independently associated with an increased likelihood of involuntary admissions beyond a primary diagnosis of psychosis, including self-harm on the emergency department record and all clinical rating scales except the DRS. Measures of dangerousness towards others were the strongest predictors among symptom measures. ${ }^{7,8,41}$ In contrast to our findings, studies in other jurisdictions have found no significant increase in $^{8,9,41}$ or lower likelihood ${ }^{7}$ of involuntary admission associated with suicide risk/self-harm, measured in this study with the SOS scale, potentially reflecting a greater focus of involuntary admissions on suicide prevention in Ontario, with dangerousness to self included as an indication. The only exception to higher likelihood of involuntary admissions with greater severity was the lower likelihood of involuntary admission with higher ratings of depression measured by the DRS, which has been found in other studies and has been suggested to reflect greater levels of insight. ${ }^{44}$ The set of factors included in this study is more diverse and extensive than that used in most previous studies, ${ }^{7,8,10,12,41,44}$ but the general finding of greater likelihood of involuntary admissions with greater severity is a consistent finding among studies.

Although we observed differences in risk factors compared with other systems, given differences in the legislation governing involuntary admissions and differences in mental healthcare systems, it is difficult to explain why risk factors may differ across systems.

\section{Conclusions}

In conclusion, we found a very high prevalence of involuntary admissions among Ontario residents that represents a substantial four-fold increase from the early 1980s. Although our data are limited in explaining the cause of the high prevalence of involuntary admissions, it is likely to be a consequence of the introduction of short involuntary holds and a dramatic reduction in the supply of psychiatric hospital beds. We found a significantly increased likelihood of involuntary admissions for individuals with police contact in the prior week, and a lower likelihood with psychiatric care, 
which is concerning given the rise in police Mental Health Act apprehensions and inadequate access to psychiatrists among those with severe mental illness. We also found increased likelihood among immigrants, even after controlling for other factors, which points to a potential disparity that requires further research to investigate. In any jurisdiction, the rights of freedom for individuals need to be balanced with the right to safety. Future research should further examine explanations for the high prevalence of involuntary admissions and determine what system-level changes may be able to mitigate this undesirable trend.

\section{Contributors}

P.K. was the Principal Investigator and with M.L. conceived the study. M.L. conducted the statistical analysis and prepared the first draft of the manuscript. All authors interpreted the data, critically revised the manuscript for important intellectual content, and approved the final version of the manuscript. M.L. had full access to all the data in the study and takes responsibility for the integrity of the data and the accuracy of the data analysis.

\section{Data sharing}

The data set from this study is held securely in coded form at the Institute for Clinical Evaluative Sciences (ICES). While data sharing agreements prohibit ICES from making the data set publicly available, access may be granted to those who meet pre-specified criteria for confidential access, available at www.ices.on.ca/DAS. The full data set creation plan and underlying analytic code are available from the authors upon request, understanding that the programs may rely upon coding templates or macros that are unique to ICES.

\footnotetext{
Michael Lebenbaum, MSc, Epidemiologist, Mental Health and Addictions Program, ICES, and PhD student, IHPME, University of Toronto, G-106, 2075 Bayview Avenue, Toronto, ON M4N 3M5; Maria Chiu, MSC, PhD, Staff Scientist, Mental Health and Addictions Program, ICES, G-106, 2075 Bayview Avenue, Toronto, ON M4N 3M5: Simone Vigod, MD, MSC, Scientist, Women's College Research Institute, Assistant Professor, IHPME, University of Toronto; Paul Kurdyak, MD, PhD, Director of Health Outcomes and Performance Evaluation (HOPE), CAMH, 250 College Street, Toronto, ON Outcomes and Performance Evaluation (HOPE), CAMH, 250 College Street, Toronto, ON
M5T 1R8, Program Lead, Mental Health \& Addictions Research Program, ICES, Associate Professor, IHPME, University of Toronto.

Correspondence: Michael Lebenbaum, Mental Health and Addictions Program, ICES, G-106, 2075 Bayview Avenue, Toronto, ON M4N 3M5, Canada.

Email: michael.lebenbaum@ices.on.ca

First received 23 May 2017, final revision 23 Aug 2017, accepted 9 Nov 2017
}

\section{Acknowledgements}

All data-sets used in the study were held securely and analysed at ICES. Parts of this material are based on data and information compiled and provided by CIHI. However, the analyses, conclusions, opinions and statements expressed herein are those of the authors, and not necessarily those of $\mathrm{CIHI}$. The authors wish to thank Immigration, Refugee and Citizenship Canada for providing data to the ICES, where the analyses were conducted. We thank Laura Holder for assistance with the analysis.

\section{Supplementary material}

Supplementary material is available online at https://doi.org/10.1192/bjo.2017.4

\section{References}

1 Newton-Howes G. Coercion in psychiatric care: where are we now, what do we know, where do we go? Psychiatrist 2010; 34: 217-20

2 Kallert TW, Glöckner M, Schützwohl M. Involuntary vs. voluntary hospital admission. A systematic literature review on outcome diversity. Eur Arch Psychiatry Clin Neurosci 2008; 258: 195-209.
3 Priebe S, Katsakou C, Glöckner M, Dembinskas A, Fiorillo A, Karastergiou A et al. Patients' views of involuntary hospital admission after 1 and 3 months: prospective study in 11 European countries. Br J Psychiatry 2010; 196: 179-85.

4 Keown P, Mercer G, Scott J. Retrospective analysis of hospital episode statistics, involuntary admissions under the Mental Health Act 1983, and number of psychiatric beds in England 1996-2006. BMJ 2008; 337: a1837.

5 Priebe S, Badesconyi A, Fioritti A, Hansson L, Kilian R, Torres-Gonzales F, et al. Reinstitutionalisation in mental health care: comparison of data on service provision from six European countries. BMJ 2005; 330: 123-6.

6 Mulder CL, Uitenbroek $D$, Broer J, Lendemeijer B, van Veldhuizen JR, van Tilburg $\mathrm{W}$, et al. Changing patterns in emergency involuntary admissions in the Netherlands in the period 2000-2004. Int J Law Psychiatry 2008; 31: 331-6.

7 Morgan C, Mallett R, Hutchinson G, Bagalkote H, Morgan K, Fearon P, et al. Pathways to care and ethnicity. 1: Sample characteristics and compulsory admission. Report from the AESOP study. Br J Psychiatry 2005; 186: 281-9.

8 Mulder CL, Koopmans GT, Selten JP. Emergency psychiatry, compulsory admissions and clinical presentation among immigrants to the Netherlands. $\mathrm{Br} \mathrm{J}$ Psychiatry 2006; 188: 386-91.

9 van der Post L, Mulder CL, Bernardt CM, Schoevers RA, Beekman AT, Dekker J. Involuntary admission of emergency psychiatric patients: report from the Amsterdam Study of Acute Psychiatry. Psychiatr Serv 2009; 60: 1543-6.

10 van der Post L, Visch I, Mulder C, Schoevers R, Dekker J, Beekman A. Factors associated with higher risks of emergency compulsory admission for immigrants: a report from the ASAP study. Int J Soc Psychiatry 2012; 58: 374-80.

11 Davies S, Thornicroft G, Leese M, Higgingbotham A, Phelan M. Ethnic differences in risk of compulsory psychiatric admission among representative cases of psychosis in London. BMJ 1996; 312: 533-7.

12 Segal SP, Laurie TA, Segal MJ. Factors in the use of coercive retention in civil commitment evaluations in psychiatric emergency services. Psychiatr Serv 2001; 52: 514-20.

13 McGarvey EL, Leon-Verdin M, Wanchek TN, Bonnie RJ. Decisions to initiate involuntary commitment: the role of intensive community services and other factors. Psychiatr Serv 2013; 64: 120-6.

14 Riley R, Richman A. Involuntary hospitalization in Canadian psychiatric inpatient facilities, 1970-1978. Can J Psychiatry 1983; 28: 536-41.

15 Archie S, Akhtar-Danesh N, Norman R, Malla A, Roy P, Zipursky RB. Ethnic diversity and pathways to care for a first episode of psychosis in Ontario. Schizophr Bull 2010; 36: 688-701.

16 Bagby RM. The effects of legislative reform on admission rates to psychiatric units of general hospitals. Int J Law Psychiatry 1987; 10: 383-94.

17 Bagby RM, Silverman I, Ryan DP, Dickens SE. Effects of mental health legislative reform in Ontario. Can Psychol 1987; 29: 21-9.

18 Malla A, Norman RM, Helmes E. Factors associated with involuntary admission to psychiatric facilities in Newfoundland. CMAJ 1987; 136: 1166-71.

19 Malla A, Norman RM. Involuntary admissions in a Canadian province: the influence of geographic and population factors. Soc Psychiatry Psychiatr Epidemiol 1988; 23: 247-51.

20 Crisanti AS, Love EJ. Characteristics of psychiatric inpatients detained under civil commitment legislation: a Canadian study. Int J Law Psychiatry 2001; 24: 399-410.

21 Tremblay PF, King PR, Baines GR. Clinical and demographic characteristics of voluntary and involuntary psychiatric inpatients. Can J Psychiatry 1994; 39: 297-9.

22 Ontario Hospital Association. A Practical Guide to Mental Health and the Law in Ontario. 2012.

23 Steele LS, Glazier RH, Lin E, Evans M. Using administrative data to measure ambulatory mental health service provision in primary care. Med Care 2004; 42: 960-5.

24 Atzema CL, Schull MJ, Kurdyak P, Menezes NM, Wilton AS, Vermuelen MJ, et al. Wait times in the emergency department for patients with mental illness. Can Med Assoc J 2012; 184: E969-E976.

25 interRAl. Scales: Status and Outcome Measures, 2017 (http://www.interrai.org/ scales.html).

26 Martin BA, Cheung KD. Civil commitment trends in Ontario: the effect of legislation on clinical practice. Can J Psychiatry 1985; 30: 259-64.

27 Czukar G, O'Brien DD. Mental Health Law in Ontario: An Overview - Final Report. 2013.

28 Hartford K, Schrecker T, Wiktorowicz M, Hoch JS, Sharp C. Report: four decades of mental health policy in Ontario, Canada. Adm Policy Ment Health Ment Health Serv Res 2003; 31: 65-73.

29 Government of Ontario. News Release: Ontario Investing \$28 Million in Mental Health and Addictions Services, 2015 (https://news.ontario.ca/mohltc/en/ 2015/02/ontario-investing-28-million-in-mental-health-and-addictions-services.html). 
30 Keown P, Weich S, Bhui KS, Scott J. Association between provision of mental illness beds and rate of involuntary admissions in the NHS in England 19882008: ecological study. BMJ 2011; 343: d3736.

31 Lamb HR, Weinberger LE, DeCuir WJ. The police and mental health. Psychiatr Serv 2002; 53: 1266-71.

32 Laura Kane. In crisis: Why are police apprehending more mentally ill people? 2014 (https://www.thestar.com/news/gta/2014/06/01/in_crisis_why_are_police_apprehending_more_mentally_ill_people.html).

33 Centre for Addiction and Mental Health. Mental Health and Criminal Justice Policy Framework. 2013

34 van der Post LF, Dekker JJ, Jonkers JF, Beekman AT, Mulder CL, de Haan L, et al. Crisis intervention and acute psychiatry in Amsterdam, 20 years of change: a historical comparison of consultations in 1983 and 2004-2005. Int J SOC Psychiatry 2010; 56: 348-58.

35 Riecher-Rossler A, Rossler W. Compulsory admission of psychiatric patients an international comparison. Acta Psychiatr Scand 1993; 87: 231-6.

36 Salize HJ, Dressing H. Epidemiology of involuntary placement of mentally ill people across the European Union. Br J Psychiatry 2004; 184: 163-8.

37 Mundt AP, Frančišković T, Gurovich I, Heinz A, Ignatyev $Y$, Ismayilov F, et al Changes in the provision of institutionalized mental health care in post-communist countries. PLOS ONE 2012; 7: e38490.
38 Bola JR, Park EH, Kim SY. Reassessing the high proportion of involuntary psychiatric hospital admissions in South Korea. Community Ment Health J 2011; 47: 603-6.

39 Zhou JS, Xiang YT, Zhu XM, Liang W, Li H, Yi J, et al. Voluntary and involuntary psychiatric admissions in China. Psychiatr Serv 2015; 66: 1341-6.

40 Steinert T, Lepping P, Baranyai R, Hoffmann M, Leherr H. Compulsory admission and treatment in schizophrenia: a study of ethical attitudes in four European countries. Soc Psychiatry Psychiatr Epidemiol 2005; 40: 635-41.

41 Wang JP, Chiu CC, Yang TH, Liu TH, Wu CY, Chou P. The low proportion and associated factors of involuntary admission in the psychiatric emergency service in Taiwan. PLOS ONE 2015; 10: e0129204.

42 Gandy HM. Form 1 - A powerful and complex tool for managing mental health problems. Paediatr Child Health 2004; 9: 222-4.

43 Hustoft K, Larsen TK, Auestad B, Joa I, Johannessen JO, Ruud T. Predictors of involuntary hospitalizations to acute psychiatry. Int J Law Psychiatry 2013; 36: $136-43$.

44 Preti A, Rucci P, Santone G, Picardi A, Miglio R, Bracco R, et al. Patterns of admission to acute psychiatric in-patient facilities: a national survey in Italy. Psychol Med 2009; 39: 485-96. 